\title{
Trial of Essential Oils to Improve Sleep for Patients in Cardiac Rehabilitation
}

\author{
Brenda McDonnell, BSN, RN, CCAP-I, PCCN-K, ${ }^{1, *}$ and Patricia Newcomb, PhD, RN, CPNP ${ }^{2}$
}

\begin{abstract}
Objectives: The objective of this study was to determine if there was a significant difference between the sleep quality of patients who inhaled placebo and those who inhaled an aroma comprising a mixture of Lavandula $x$ intermedia (Lavandin Super), Citrus bergamia (bergamot), and Cananga odorata (ylang ylang).

Design: This was a randomized, double-blind crossover study, which compared a treatment with placebo.

Settings/location: The study was conducted in an outpatient cardiac rehabilitation unit located in an urban, private nonprofit hospital in north Texas.

Subjects: Participants included 42 adult patients referred to the rehabilitation service following hospitalizations by local cardiologists.

Interventions: Cotton balls infused with a combination of lavender, bergamot, and ylang ylang or placebo were placed at subjects' bedsides for five nights. After a washout period, subjects crossed over to the other condition for five nights.

Outcome measures: Participants completed the Pittsburgh Sleep Quality Index (PSQI) after treatment and placebo periods. Lower PSQI scores indicate better sleep quality.

Results: The mean PSQI global score was statistically significantly lower when receiving the intervention oil $($ mean $=4.9)$ than the mean PSQI global score when receiving placebo $($ mean $=8)$. Duration of sleep and the time it took to fall asleep were no different between treatment groups, but patient-reported sleep quality was significantly better when participants were exposed to essential oils $\left(\chi^{2}=4.5, p=0.03\right)$ than when exposed to placebo. Although sleep efficiency (time asleep while lying in bed) was perceived as the same under both conditions, participants reported that they had to get up to use the bathroom significantly less often when exposed to the treatment than when exposed to placebo $(t=-2.04, p=0.05$; Wilcoxon $p=0.05)$. Participants also reported that they had trouble sleeping because they felt too cold, which occurred significantly less often when exposed to the treatment than when exposed to placebo $(t=-2.03, p=0.05$; Wilcoxon $p=0.05)$.

Conclusions: Sleep quality of participants receiving intervention oils was significantly better than the sleep quality of participants receiving the placebo oil. Low-cost, nontraditional aromatherapy treatment is potentially effective for improving sleep quality among cardiac rehabilitation patients.
\end{abstract}

Keywords: sleep, essential oils, cardiac rehabilitation, lavender, bergamot, ylang ylang

\section{Introduction}

Leep Disturbances have BeEn linked to high blood pressure, atherosclerosis, heart failure, heart attack, diabetes, stroke, and obesity. Poor sleep triggers the body to produce stress hormones that can contribute to cardiovascular disease. ${ }^{1}$ Drugs, such as hypnotics and benzodiazepines, are frequently prescribed to help heart failure patients sleep, but the adverse effects of such therapy can be substantial. ${ }^{2}$ Evidence shows that aromatherapy can be an inexpensive, noninvasive complementary approach to promote quality sleep in hospital settings. ${ }^{3,4}$ Patients in home settings may experience

\footnotetext{
${ }^{1}$ Cardiac Rehabilitation Program, Texas Health Harris Methodist Hospital Fort Worth, Fort Worth, TX.

${ }^{2}$ Nursing Operations, Texas Health Resources, Arlington, TX.

*Current affiliation: Medical-Surgical Nursing, Texas Health Harris Methodist Alliance, Fort Worth, TX.
} 
similar benefits, as shown by Hwang and Shin who concluded in their systematic review and meta-analysis that inhaled aromatherapy significantly improves sleep, especially in unhealthy individuals. ${ }^{5}$

The chemical composition of certain essential oils can plausibly be associated with sedative and hypnotic effects. ${ }^{6}$ Esters and alcohols are two of the chemical functional groups that promote calming and tranquil therapeutic effects, resulting in reduced anxiety and enhanced sleep quality. ${ }^{7}$ For instance, linalool is an alcohol molecule that acts as a hypnotic by inhibiting the chemical binding of glutamate receptors in the cerebral cortex of the brain. Glutamate receptors are responsible for glutamate-mediated postsynaptic excitation of neural cells. When linalool inhibits glutamate binding, neural excitation is reduced and sedative as well as potential neuroprotective effects result. ${ }^{8-10}$ Linalool is a component of numerous essential oils, including lavender, which has been shown to have soporific effects in animal and human studies. ${ }^{11-13}$

Esters are formed when an organic acid and an alcohol combine. Even though the method of action for esters is not fully understood, they are considered aromatically and therapeutically desirable. Esters are commonly accepted in the aromatherapy community as regulators of the nervous system, and essential oils that contain esters are thought to be most relaxing, balancing, and calming. ${ }^{14}$ Benzyl acetate, an ester of benzyl alcohol and acetic acid, is a significant ester found in ylang ylang. The acetate ester of linalool, linalyl acetate, is classified as an antimicrobial and flavoring agent and occurs in bergamot and lavender. ${ }^{15} \mathrm{~A}$ third ester of interest here, geranyl acetate, is also classified as a flavoring agent and is found in lavender. ${ }^{16}$ Geranyl acetate has the interesting effect of masking the perception of floral aroma, which may, in addition to any sedative effect it may exert, help make the odors associated with essential oil mixtures more tolerable. ${ }^{17}$

Results of epidemiological studies support the claim that poor quality sleep influences cardiovascular morbidity, and some randomized trials using essential oils as sleep aids have been reported. The chemistry of certain constituents in selected essential oils indicates plausible sedative effects of the oils. Given the few trials that exist and biochemistry of the oils, the primary objective of this study was to determine whether an essential oil mixture improves sleep quality in cardiac patients. The authors hypothesized that patients who inhaled an aroma comprising essential oils high in linalool and esters would experience better sleep quality than patients who were not exposed.

\section{Materials and Methods}

\section{Setting and sample}

This study employed a sample of participants from an urban hospital's outpatient cardiac rehabilitation unit. Institutional Review Board (IRB) approval was obtained, and recruitment took place through flyers and personal communication between patients enrolled in the cardiac rehabilitation program and program nurses. During the study period, 101 patients were enrolled in the cardiac rehabilitation program and were informed about the study. Those with asthma and nut allergies were excluded to minimize the risk of reactions to essential oils. Other interested partici- pants over the age of 18, who spoke English, and provided consent were included in the study. As shown in Figure 1, 50 patients consented. Five completed only half of the study, with three withdrawing because they found the aroma of the oils disturbing. The total number of participants with complete data for analysis was 42 . A priori power analysis indicated that a total sample of 20 provided power of 0.85 , assuming an $\alpha$ of 0.05 and mean difference in scores of at least $1 .{ }^{18}$ Twelve females and 30 males between the ages of 43 and 85 were included. Participants' referring doctor or primary care physician was notified of patient participation in the study.

A patient advisory board consisting of two patients from cardiac rehabilitation, a male and a female, provided suggestions regarding the processes of study implementation. They were asked to serve because of their long-term rehabilitation participation and their commitment to being available for the duration of the study. A safety committee was formed to evaluate any participant-reported adverse events. Safety committee members included nurses who were certified aromatherapists, but were not part of the research team. No adverse events were reported.

\section{Research design}

This was a randomized, double-blind crossover study that examined 42 participants from an urban, outpatient cardiac rehabilitation unit. Figure 2 illustrates the study design. Packets were prepared for subjects to take to their homes. Packets contained cotton balls infused with either intervention oils or placebo oil. Intervention oils consisted of equal parts of Lavandula $x$ intermedia (Lavandin Super), Citrus bergamia (bergamot), and Cananga odorata (ylang ylang). Lavandula $x$ intermedia, rather than the more commonly used Lavandula angustifolia, was chosen because at least one previous study found Lavandula x intermedia to be almost twice as effective as $L$. angustifolia in inducing relaxation among cardiac patients. ${ }^{19}$

Intervention oils were obtained from Nature's Gift. Oils were organic and of medical grade. The company supplied

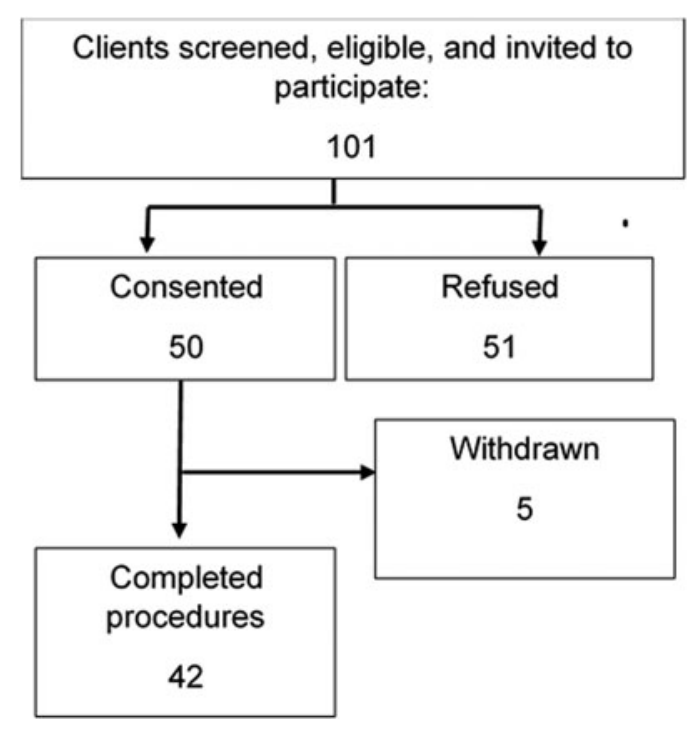

FIG. 1. Recruitment. 


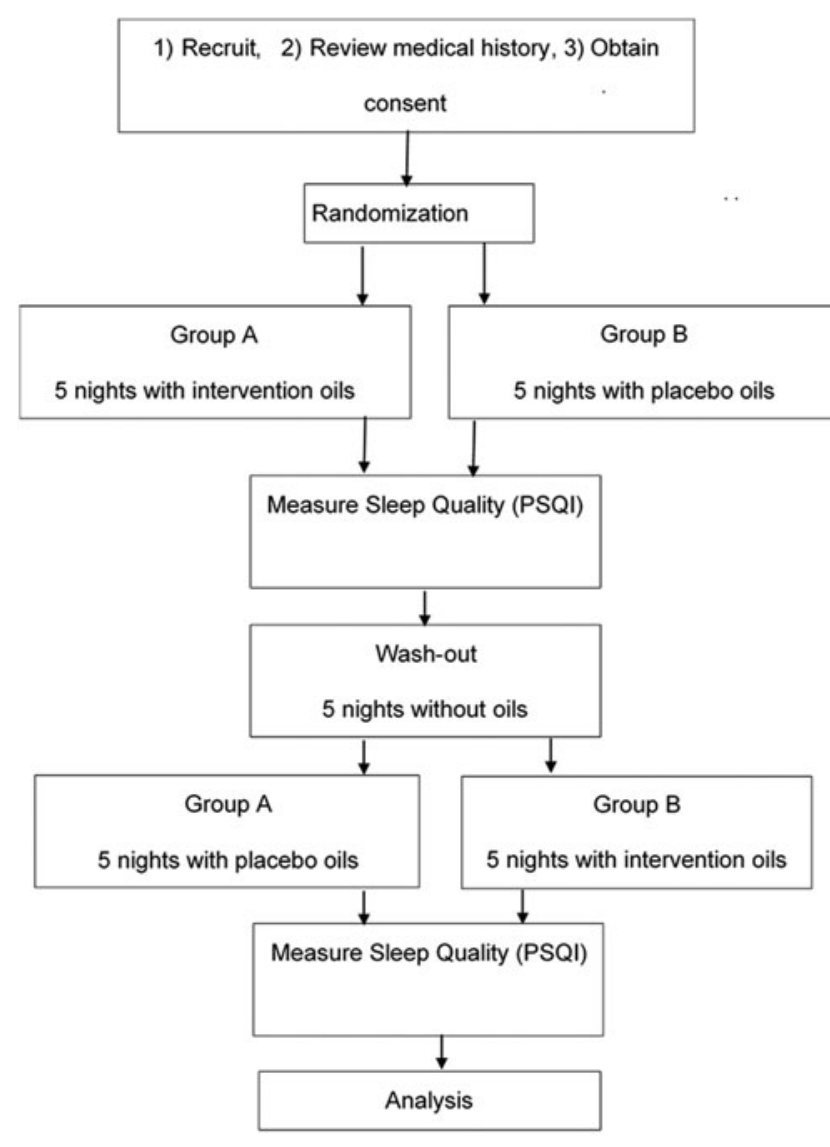

FIG. 2. Study design. tained jojoba oil, and three cotton balls were placed in the mesh cloth bag. The mesh bag was then placed in a resealable plastic bag. Both intervention and placebo oils had a yellow color that was muted by the black color of the mesh cloth bag. Although odors of the placebo and essential oil preparations were different, they were vague enough to not be identifiable. Subjects were aware that both treatments had odors, but claimed to be unable to distinguish which odors were produced by which oils. Instructions for use, as shown in Figure 3, were placed inside each packet. Packets were prepared by the primary investigator, a certified aromatherapist, but were randomized by a member of the research team (P.N.) who did not have contact with patients to preserve the double-blind nature of the study.

Subjects placed cotton balls infused with the intervention oils or placebo oil at their bedside for five nights. At the end of the 5-night study period, participants completed a self-report measure of sleep quality, the Pittsburgh Sleep Quality Index (PSQI). After a 1-week washout period, participants switched to the opposing group and repeated the treatment procedure and measure. Bedside referred to any surface 6-36 inches from the subject's pillow. Many participants hung their mesh bags on a bedpost and others placed it on a bedside table. Adherence was monitored by means of verbal self-report from subjects at the end of each treatment week.

Polysomnography is the gold standard to measure sleep characteristics. The study setting (patient homes) and resources did not permit polysomnography. Instead, a selfreport measure, the PSQI, was used. The PSQI is the most commonly used tool in the United States to measure sleep gas chromatography patterns of all oil batches, ${ }^{20-22}$ showing the proportions of linalool and esters in each oil, as shown in Table 1. One drop of each undiluted oil was applied to a cotton ball that contained jojoba oil and three cotton balls were placed in a mesh cloth bag, then placed in a resealable, ziplock plastic bag. The scent of the resulting mixture was mild, but not distinctive. Subjects were unable to identify components of the mixture by odor, which was considered an important qualification of the treatment given that there are many body creams and other preparations containing lavender currently on the market.

Placebo cotton balls were infused with walnut oil, which has a mild scent, which is also not distinctive. The placebo oil was purchased over the counter from Kroger grocery and contained $100 \%$ walnut oil made by La Tourangelle. To be consistent with the intervention group, three drops of $100 \%$ walnut oil were applied to each cotton ball, all balls con-

Table 1. Proportions of Target Substances IN STUdy OILs

\begin{tabular}{lccc}
\hline & \multicolumn{3}{c}{ Study oils (\%) } \\
\cline { 2 - 4 } $\begin{array}{l}\text { Target } \\
\text { substance }\end{array}$ & $\begin{array}{c}\text { Lavandula } \mathrm{x} \\
\text { intermedia super }\end{array}$ & $\begin{array}{c}\text { Citrus } \\
\text { bergamia }\end{array}$ & $\begin{array}{c}\text { Cananga } \\
\text { odorata }\end{array}$ \\
\hline Linalool & 37.12 & 10.63 & 6.26 \\
Esters & 30.37 & 30.12 & 57.93 \\
\hline
\end{tabular}

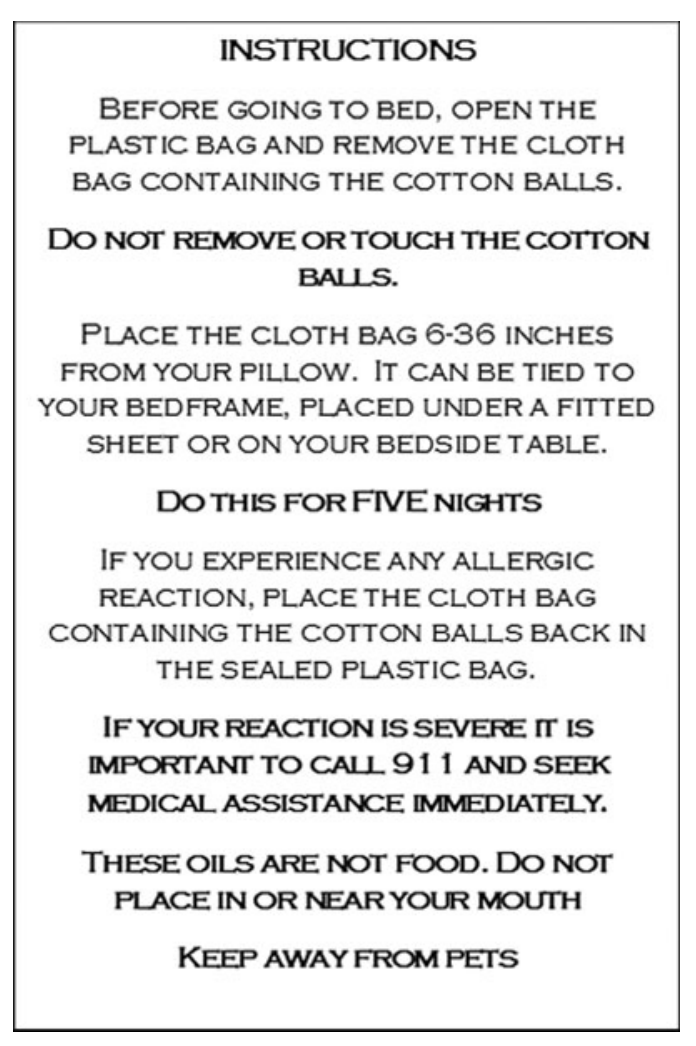

FIG. 3. Instructions for subjects. 
quality and there is evidence to support the tool's validity and reliability in numerous studies. ${ }^{23}$ It contains 19 questions grouped into 7 subtests and yields a global score of 0 21 , where 0 indicates no difficulty sleeping and a score $>5$ indicates poor quality sleep, thus lower PSQI global scores indicate better sleep quality. A PSQI improvement of three points on the global score is considered a substantial improvement in sleep quality. ${ }^{24,25}$ Permission was obtained from the University of Pittsburgh to use a modified version of the PSQI that included the term "one week" in the instructions rather than "one month," thus subjects were asked to recall sleep characteristics for the past week.

\section{Data analysis}

SPSS IBM Statistical Software Package, version 20, was used to compile and analyze the collected data. The primary outcome was a global PSQI score. Mean PSQI scores are presented visually in a boxplot and compared by means of a correlated $t$-test. Since distributions of global scores were not perfectly normal (skewed toward better sleep quality), a related samples Wilcoxon signed-rank test was also run as a check on the $t$-test. Chi-square was used to compare selected nominal variables. Age and gender were statistically controlled by means of linear regression.

Per-protocol analysis was employed. Intention-to-treat analysis was not planned due to the fact that all subjects participated in both interventions by design and because follow-up was not planned for subjects who withdrew from the study, which was expected to result in missing outcome data.

\section{Results}

Fifty subjects participated in the trial. Age of participants ranged from 45 to 85 years with a mean of 67 years. Twelve participants were female and 37 were male. Most participants (66\%) reported going to bed between 10 and 11 PM. At baseline, 57\% of participants reported that they fell asleep within $15 \mathrm{~min}$ of going to bed and only $9 \%$ reported lying awake for more than half an hour before falling asleep. However, $50 \%$ of the sample reported that they woke up in the middle of the night or too early in the morning on at least
3 days during the week before assessment. Frequencies of responses for PSQI items regarding sleep challenges are presented in Table 2.

Internal consistency reliability of scaled items on the PSQI (Cronbach's $\alpha=0.78$ ) was slightly lower than the 0.83 reported in a previous study, ${ }^{24}$ but still acceptable. The mean PSQI global score when receiving the intervention oil was 4.9, and the mean PSQI global score when receiving placebo was 8 (Fig. 4). Global PSQI scores during treatment with essential oils were significantly better than global scores during treatment with placebo $(t=-6.386, p=0.0001)$. Results of the $t$-test are shown in Table 3 and are consistent with results of the Wilcoxon signed-rank test $(p=0.0001)$. Age and gender demonstrated no significant influence when regressed on global PSQI scores.

Duration of sleep and the time it took to fall asleep were no different between treatment groups, but patient-reported sleep quality on a single item, "How would you rate your sleep quality overall during the past week?" was significantly better when participants were exposed to the intervention $\left(\chi^{2}=4.5, p=0.03\right)$ than when exposed to placebo. Although sleep efficiency (time asleep while lying in bed) was perceived similarly under both conditions, participants reported that they had to get up to use the bathroom significantly less often when exposed to the treatment than when exposed to placebo $(t=-2.04, p=0.05$; Wilcoxon $p=0.05$ ). Participants also reported that they had trouble sleeping because they felt too cold, which occurred significantly less often when exposed to the treatment than when exposed to placebo $(t=-2.03, p=0.05$; Wilcoxon $p=0.05)$.

\section{Discussion}

Although initial insomnia did not bother most participants, $50 \%$ of the sample reported difficulty remaining asleep during the night and early morning due to sleep disruptions related to the need to use the bathroom and to feelings of being chilled. This represents a substantially higher rate of disturbed sleep than apparent from general insomnia statistics in previous literature, which typically reports about a third of individuals in developed nations to be sleep disturbed. ${ }^{26-28}$ Previous studies of individuals with

Table 2. Mean Scores for Responses to Sleep Characteristics-Items of Pittsburgh Sleep QuAlity Index Section 5

\begin{tabular}{lccc}
\hline \multicolumn{4}{c}{ During the past week, how often have you had trouble sleeping because you..... } \\
\hline & $\begin{array}{c}\text { Intervention } \\
\text { mean (SD) }\end{array}$ & $\begin{array}{c}\text { Control } \\
\text { mean (SD) }\end{array}$ & $\begin{array}{c}\text { Significance } \\
\text { of difference }\end{array}$ \\
& $0.66(0.987)$ & $0.93(1.136)$ & Nonsignificant \\
(a) cannot get to sleep within 30 min & $2.14(1.002)$ & $2.07(1.176)$ & Nonsignificant \\
(b) wake up in the middle of the night or early morning & $2.18(1.126)$ & $2.42(1.011)$ & 0.046 \\
(c) have to get up to use the bathroom & $0.11(0.493)$ & $0.13(0.548)$ & Nonsignificant \\
(d) cannot breathe comfortably & $0.41(0.996)$ & $0.47(1.014)$ & Nonsignificant \\
(e) cough or snore loudly & $0.20(0.553)$ & $0.44(0.918)$ & 0.046 \\
(f) feel too cold & $0.50(0.902)$ & $0.69(1.019)$ & Nonsignificant \\
(g) feel too hot & $0.25(0.534)$ & $0.42(0.917)$ & Nonsignificant \\
(h) had bad dreams & $0.61(1.039)$ & $0.62(1.114)$ & Nonsignificant \\
(i) have pain & & &
\end{tabular}

Possible scores ranged from 0 (not during the past week) to 3 ( $\geq 3$ days during the past week).

${ }^{a}$ Related samples Wilcoxon signed-rank test.

$\mathrm{SD}$, standard deviation. 


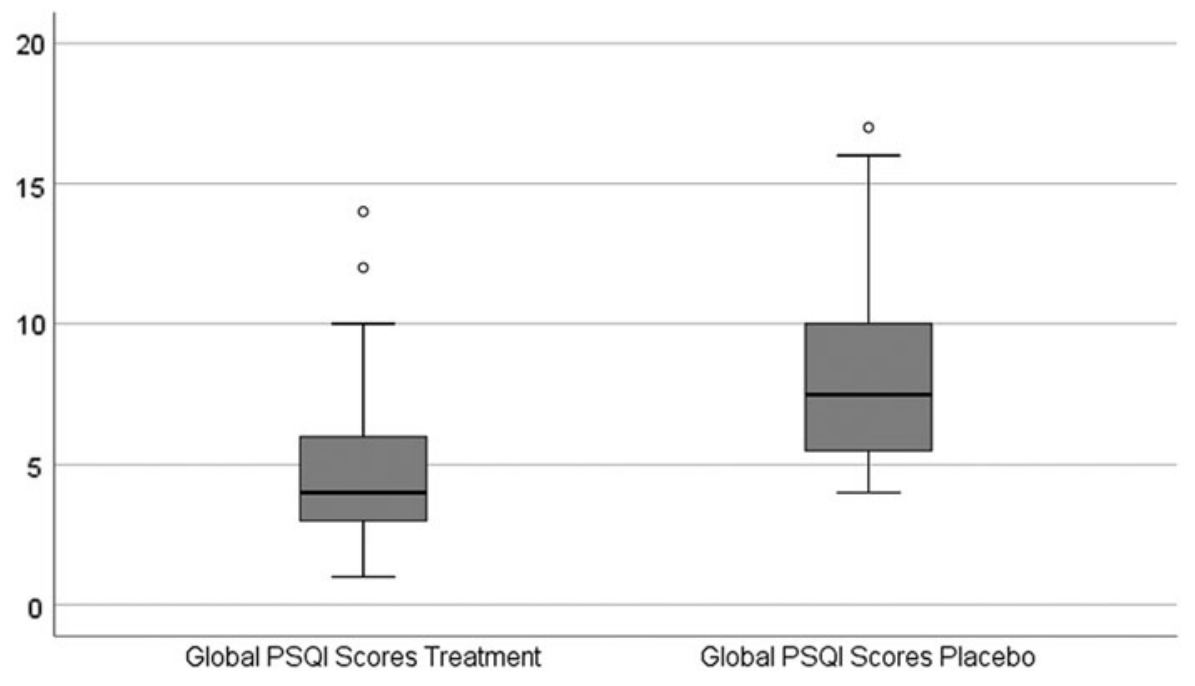

FIG. 4. PSQI mean scores for intervention and placebo groups. PSQI, Pittsburgh sleep quality index. heart failure found higher rates of sleep disturbances than in the general population, and their findings (40\%-45\%) are more consistent with the findings here, ${ }^{29-31}$ highlighting the importance of assessing sleep and addressing sleep problems in cardiac rehabilitation efforts.

The effect of essential oil inhalation on PSQI scores was substantial in this study (effect size: $d=1$ ). This finding is consistent with earlier trials using lavender oil as the intervention and PSQI as a measure of sleep quality. $4,24,32-34$ PSQI results of trials of bergamot or ylang ylang singly or in combination with other oils on sleep quality were not found in the authors' literature search. Much of the work on lavender and sleep has been published by nurses and emphasizes the point that treatment with essential oils is an intervention within the scope of practice of most nurses and is inexpensive enough to be used in resource-poor populations.

Results of this study also indicated that the essential oil combination used in this project influenced the quality of sleep rather than sleep duration. The fact that participants experienced less sleep disruption as a result of fewer bathroom visits and reported a more comfortable perceived temperature during exposure to essential oils suggests that subjects may be sleeping more deeply when exposed to essential oils. A potential hypothesis that emerges from this line of thought is that inhalation of intervention oils promotes more time spent in stage 3 non-rapid eye movement (REM) sleep, the stage of the sleep cycle that is critical for deep

TAble 3. T-Test For Differences in MeAns of Global Pittsburgh Sleep Quality Index Scores

\begin{tabular}{lcc}
\hline & Essential oil treatment & Placebo \\
\hline Mean PSQI score & 4.9 & 8 \\
$t$-Value for difference & -6.386 & \\
$p$-Value for difference & 0.0001 \\
Effect size (Cohen's d) & 1.00 (very large effect) \\
Mean difference & 3.1 \\
CI of the difference & $2.12-4.08$
\end{tabular}

Validated with related samples Wilcoxon signed-rank test, $p=0.000$.

CI, confidence interval; PSQI, Pittsburgh sleep quality index. restorative sleep. This interpretation is consistent with findings from two previous studies. Goel et al. found that the scent of lavender increased the percentage of slow-wave (non-REM) sleep in subjects, as measured by polysomnography, ${ }^{8}$ and Perl et al. found that odors presented during sleep, including lavender among others, enhanced slow-wave activity during non-REM sleep. ${ }^{34}$ Since stage 3 non-REM sleep diminishes with aging, discovery of ways to safely support and prolong this sleep stage in elders could be of great utility; however, further interest in studying the relationship between inhalation of traditional essential oils and changes in stages of human sleep has been lacking. This suggests that there are interesting opportunities for future research on the effects of essential oils (including oils other than lavender) on specific sleep stages among children, young adults, and older adult cardiac patients specifically, as well as cardiac patients generally.

Sleeping medications typically prescribed for cardiac patients to use at home include medications such as zolpidem, eszopiclone, and diphenhydramine. Adverse effects associated with zolpidem can be dramatic, including hallucinations, anxiety, memory impairment, and other central nervous system manifestations. In addition, zolpidem can cause cardiovascular effects, such as chest pain, blood pressure changes, edema, and tachycardia, thus making it a poor choice for cardiac patients. Eszopiclone has similar effects, and diphenhydramine is discouraged for use among older adults. Given the safety and lack of expense for oils, inhaling essential oils for improving sleep should be considered as an alternative to the hypnotics and antihistamines frequently prescribed for home use today.

\section{Limitations and suggestions for future research}

Attrition was experienced in the study, including five subjects who formally withdrew and an additional three who did not complete one or more procedures. This may have resulted in reduced power, thus although the effects described above were discernable, others may have been missed. No follow-up for withdrawn subjects was conducted. This is not untypical of studies conducted using consumers as subjects in clinical environments. Companies 
have a valid concern since collecting data following withdrawal could be construed to constitute failure to genuinely withdraw a patient from the study or maintaining participation without consent. Opinions may vary among IRBs.

In this study, a self-report tool was used to measure sleep quality. The PSQI is a well-established tool with good evidence for reliability and validity, but it is subjective. Using polysomnography as an objective tool to measure sleep quality would improve the evidence regarding the relationship between essential oils and sleep, including effects on sleep stage. Due to labor and time constraints, the only selfreport measure possible in this project was the PSQI; however, in future, research measuring and controlling for depression and anxiety would be relevant. Comparing the effectiveness of essential oils for sleep between ambulatory populations and patients hospitalized for acute care would be of interest as well. Rigorous research on effects of different combinations of inhaled essential oils containing high amounts of linalool and esters and recruiting more diverse samples would be worthwhile as well. It would also be of interest to compare effects of each of the target essential oils singly with effects of the combination used in this project.

Keeping infused cotton balls in plastic containers between uses and limiting use to five nights were factors included in the study design to control for essential oil freshness, but fragrance concentration and its degradation over time were not measured specifically. Fragrance concentration could be an important factor in an aromatherapy intervention, but, lacking equipment to measure fragrance concentration, the authors chose to employ products that would be easily accessible and easily used by anyone in a home setting. In any case, evidence regarding the correlation of fragrance concentration and fragrance perception is lacking, thus the human nose may be the best measure of perceived odors. In this study, no subject reported that fragrances diminished perceptibly over the week of treatment and the researchers detected no changes, but the question points to opportunities for future research.

\section{Conclusions}

The sleep quality of patients enrolled in an outpatient cardiac rehabilitation program was significantly better when patients inhaled a mixture of essential oils at night than when they inhaled placebo. Evidence from this study suggests that inhaling a mixture of essential oils can provide a natural, inexpensive, and safe approach to help cardiac patients gain better rest.

\section{Acknowledgment}

The authors would like to thank Huy Nguyen, MS, Lake Erie College of Osteopathic Medicine at Seton Hill, Greensburg, PA, for his help as a research assistant while interning during his master's program at University of North Texas, Fort Worth, TX.

\section{Author Disclosure Statement}

No competing financial interests exist.

\section{Funding Information}

This was an unfunded project made possible by labor and in-kind support from Texas Health Resources.

\section{References}

1. The Society for Cardiovascular Angiography and Interventions (SCAI) [homepage on the Internet]. Sleep and cardiovascular disease. 2015. Online document at: www.secondscount.org/ healthy-living/healthy-living-detail?cid=828f98c1-fcb1-4e038af3-2e433847be3f\#.VTPFHBt0yU, accessed February 14, 2019.

2. Sateia MJ, Buysse DJ, Krystal AD, et al. Clinical practice guidelines for the pharmacologic treatment of chronic insomnia in adults: An American Academy of Sleep Medicine clinical practice guideline. J Clin Sleep Med 2017;13: 307-349.

3. Ayik C, Özden D. The effects of preoperative aromatherapy massage on anxiety and sleep quality of colorectal surgery patients: A randomized controlled study. Complement Ther Med 2018;36:93-99.

4. Lytle J, Mwatha C, Davis KK. Effect of lavender aromatherapy on vital signs and perceived quality of sleep in the intermediate care unit: A pilot study. Am J Crit Care 2014; 23:24-29.

5. Hwang E, Shin S. The effects of aromatherapy on sleep improvement: A systematic literature review and metaanalysis. Altern Complement Med 2015;21:61-68.

6. Buckle J. Clinical Aromatherapy Essential Oils in Healthcare (3rd Ed). St. Louis, MO: Elsevier, 2015.

7. Swanson TA, Kim SI, Glucksman MJ. Biochemistry, Molecular Biology \& Genetics. Baltimore, MD: Lippincott Williams \& Wilkins. 2010.

8. Goel N, Kim H, Lao RP. An olfactory stimulus modifies nighttime sleep in young men and women. Chronobiol Int 2005;22:889-904.

9. Rezaee R, Hosseinzadeh H. Safranal [homepage on the Internet]. From an aromatic natural product to a rewarding pharmacological agent. US National Library of Medicine National Institutes of Health. 2013. Online document at: www .ncbi.nlm.nih.gov/pmc/articles/PMC3637901/, accessed May 21, 2015.

10. Sabogal-Guaqueta AM, Posada-Duque R, Cortes NC, et al. Changes in the hippocampal and peripheral phospholipid profiles are associated with neurodegeneration hallmarks in a long-term global ischemia model: Attenuation by linalool. Neuropharmacology 2018;135:555-571.

11. Baldisserotto B, Barata L, Silva AS, et al. Anesthesia of tambaqui Colossoma macropomum (Characiformes: Saerrasalmidae) with the essential oils of Aniba rosaeodora and Aniba parviflora and their major compound, linalool. Neotrop ichthyol 2018;16:1-6.

12. Takeda A, Watanuki E, Koyama S. Effects of inhalation aromatherapy on symptoms of sleep disturbance in the elderly with dementia. J Evid Based Complementary Altern Med 2017;2017:1902807.

13. Ali B, Al-Wabe NA, Shams S, et al. Essential oils used in aromatherapy: A systematic review. Asian Pac J Trop Dis 2015;5:601-611.

14. Stewart D. The Chemistry of Essential Oils Made Simple. Marble Hill, MO: Care Publications, 2005.

15. ChEBI (Chemical Entities of Biological Interest) [homepage on the Internet]. CHEBI:6469-linalyl acetate. 2018. Online document at: https://www.ebi.ac.uk/chebi/chebiOntology .do?chebiId=CHEBI:6469, accessed February 14, 2019.

16. National Center for Biotechnology Information [homepage on the Internet]. PubChem Compound; CID $=1549026$. Online document at: https://pubchem.ncbi.nlm.nih.gov/compound/ geranyl_acetate\#section=Top, accessed February 27, 2019. 
17. Xiao Z, Luo J, Niu Y, et al. Olfactory impact of esters on rose essential oil floral alcohol aroma expression in model solution. Food Res Int 2019;116:211-222.

18. Hintz $\mathrm{J}$ [homepage on the Internet]. NCSS, PASS, and GESS Software. Kaysville, UT: NCSS. 2006. Online document at: www.NCSS.com, accessed September 6, 2019.

19. Buckle J. Does it matter which lavender essential oil is used? Nursing Times 1993;89:32-35.

20. Clark $M$ [homepage on the Internet]. Bergamot. In: Nature's Gift. 2015. Online document at: http://naturesgift.com/ resources/gc/bergamot-organic-italy-jun2014-IT.B120027-gc .pdf, accessed September 6, 2019.

21. Clark $M$ [homepage on the Internet]. Lavandin super. In: Nature's Gift. 2015. Online document at: http://naturesgift .com/resources/gc/lavandin-super-organic-france-2014-gc .pdf, accessed September 6, 2019.

22. Clark M [homepage on the Internet]. Ylang ylang. In: Nature's Gift. 2015. Online document at: http://naturesgift.com/ resources/gc/ylangylang-complete-organic-madagascar-jun 2014-MAB975070-gc.pdf, accessed September 6, 2019.

23. Buysse DJ, Reynolds CF, Monk TH, et al. The Pittsburgh sleep quality index: A new instrument for psychiatric practice and research. Psychiatry Res 1989;28;193-213.

24. Lewith GT, Godfrey AD, Prescott P. A single-blinded, randomized pilot study evaluating the aroma of lavandula augustifolia as a treatment for mild insomnia. Altern Complement Med 2005;11:631-637.

25. Smyth $\mathrm{C}$ [homepage on the Internet]. The Pittsburgh sleep quality index (PSQI). 2012. Online document at: https:// consultgeri.org/try-this/general-assessment/issue-6.1.pdf, accessed September 6, 2019.

26. Mellor A, Hamill K, Jenkins MM, et al. Partner-assisted cognitive behavioral therapy for insomnia versus cognitive behavioral therapy for insomnia: A randomized controlled trial. Trials 2019;20:262.

27. Van de Straat V, Bracke P. How well does Europe sleep? A cross-national study of sleep problems in European older adults. Int J Public health 2015;60:643-650.
28. Centers for Disease Control and Prevention [homepage on the Internet]. Sleep and sleep disorders. 2018. Online document at: https://www.cdc.gov/sleep/index.html, accessed June 7, 2019.

29. Johansson P, Brostrom A, Sanderman R, Jaarsma T. The course of sleep problems in patients with heart failure and associations to rehospitalizations. J Cardiovasc Nurs 2015; 30:403-410.

30. Edwards KS, Hoover V [homepage on the Internet]. Insomnia and heart disease. American College of Cardiology. 2016. Online document at: https://www.acc.org/latest-incardiology/articles/2016/08/02/07/25/insomnia-and-heartdisease, accessed June 7, 2019.

31. Karadag E, Samancioglu S, Ozden D, et al. Effects of aromatherapy on sleep quality and anxiety of patients. Nurs Crit Care 2017;22:105-112.

32. Moeini M, Khadibi M, Bekhradi R, et al. Effect of aromatherapy on the quality of sleep in ischemic heart disease patients hospitalized in intensive care units of heart hospitals of the Isfahan University of Medical Sciences. Iran J Nurs Midwifery Res 2010;15:234-239.

33. Faydali S, Cetinkaya F. The effect of aromatherapy on sleep quality of elderly people residing in a nursing home. Holist Nurs Pract 2018;32:8-16.

34. Perl O, Arzi A, Sela L, et al. Odors enhance slow-wave activity in non-rapid eye movement sleep. J Neurophysiol 2016;115:2294-2302.

Address correspondence to: Patricia Newcomb, PhD, RN, CPNP

Nursing Operations

Texas Health Resources

612 E. Lamar Boulevard Arlington, TX 76011-4133

E-mail: patricianewcomb@texashealth.org 